\title{
Potential Mechanisms of Overtraining
}

\author{
Jeremy Park, Lorin E. Donovan, \& Joon Young Kim \\ Syracuse University, New York, USA
}

\begin{abstract}
Frequent/intense training increases the risk of overtraining in athletes. When overtrained, despite high effort in training, performance stagnates and/or deteriorates (Cadegiani \& Kater, 2018). A growing body of literature has attempted to decipher the pathophysiology of overtraining. However, the majority of these literatures focus on the metabolic, biochemical, hormonal, and immunological aspects of overtraining. Few studies have analyzed more applicable biomarkers of overtraining such as eating and sleeping patterns, body composition, mood, and libido with conclusive results. A previous study entitled "Endocrine and Metabolic Responses on Overtraining Syndrome (EROS)" has attracted researchers' attention as they measured relevant/applicable biomarkers for overtraining. The purpose of the study by Cadegiani and Kater (2018) is to elaborate the EROS study and understand the mechanisms of overtraining more comprehensively.
\end{abstract}

Keywords: Type 2 diabetes risk, glucose response curve, 1-hr glucose concentration, lifestyle intervention

\section{Article History}

Received 18 September 2020

Accepted 11 October 2020

Published 31 January 2021

Available online 19 February 202

https://doi.org/10.47544/johsk.2021.2.1.15
Corresponding Author

Joon Young Kim

jkim291@syr.edu

Department of Exercise Science

The David B. Falk College of Sport and Human Dynamics Syracuse University, USA

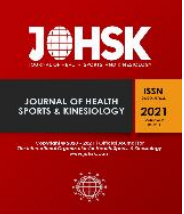

\section{Methods}

Male volunteers (age 18-50 years) were screened based on health history, drug use, exercise patterns, and overtraining symptoms (i.e., unexplained performance decreases). After prescreening, selected participants $(n=51)$ were further tested on their sleeping, eating, and social patterns (self-reported), and psychological state by the POMS (Profile of Mood States) questionnaire. Based on these answers, participants were divided into three groups: healthy athletes (ATL), overtrained athletes (OTS), and non-physically active controls (NCS). Using bioimpedance and air-displacement, body hydration and composition were measured. Daily caloric/macronutrient intake, POMS, time spent studying/working (hours/day), libido on a scale 1-10, and basal metabolic rate (BMR) by indirect calorimetry were also measured for analysis.

\section{Results}

Compared to ATL, OTS reported reduced relative calorie intake $(26.4 \mathrm{kcal} / \mathrm{kg} / \mathrm{day}$ in OTS vs. $52.7 \mathrm{kcal} / \mathrm{kg} / \mathrm{day}$ in ATL), worse sleep quality on a scale 1-10 (6.5 vs. 8.0), worse mood (POMS scores of 15.0 vs. 5.0 on anger, 5.0 vs. 2.0 on confusion, 7.5 vs. 0.0 on depression, 20.0 vs. 2.0 on fatigue, 16.5 vs. 6.0 on tension, and 9.5 vs. 26.0 vigor), lower measured-to-predicted BMR (100.5\% vs. 109.0\%), higher body fat (15.3\% vs. $10.3 \%)$, and diminished libido on a scale

\section{Journal of Health, Sports, \& Kinesiology | ISSN 2692-9864 | www.johsk.com}


1-10 (7.0 vs. 8.0) and hydration (60.8\% vs. $64.9 \%$ body water) (all $P<0.05)$. OTS had greater musculature than NCS (40.2 kg vs. $33.7 \mathrm{~kg}$ ) and spent more time studying/working than ATL (8 vs. 7 hours/day) (all P<0.05).

\section{Discussion}

Declining performance observed in OTS may originate from inadequate sleep, which prevents optimal reparative processes. Disproportionally high body fat may have caused reduced BMR in OTS. Excessive working/studying may impair physical performance as cognition requires energy. Critical risk factors for overtraining are likely inadequate sleep, excessive cognition, and insufficient caloric intake (Figure 1).

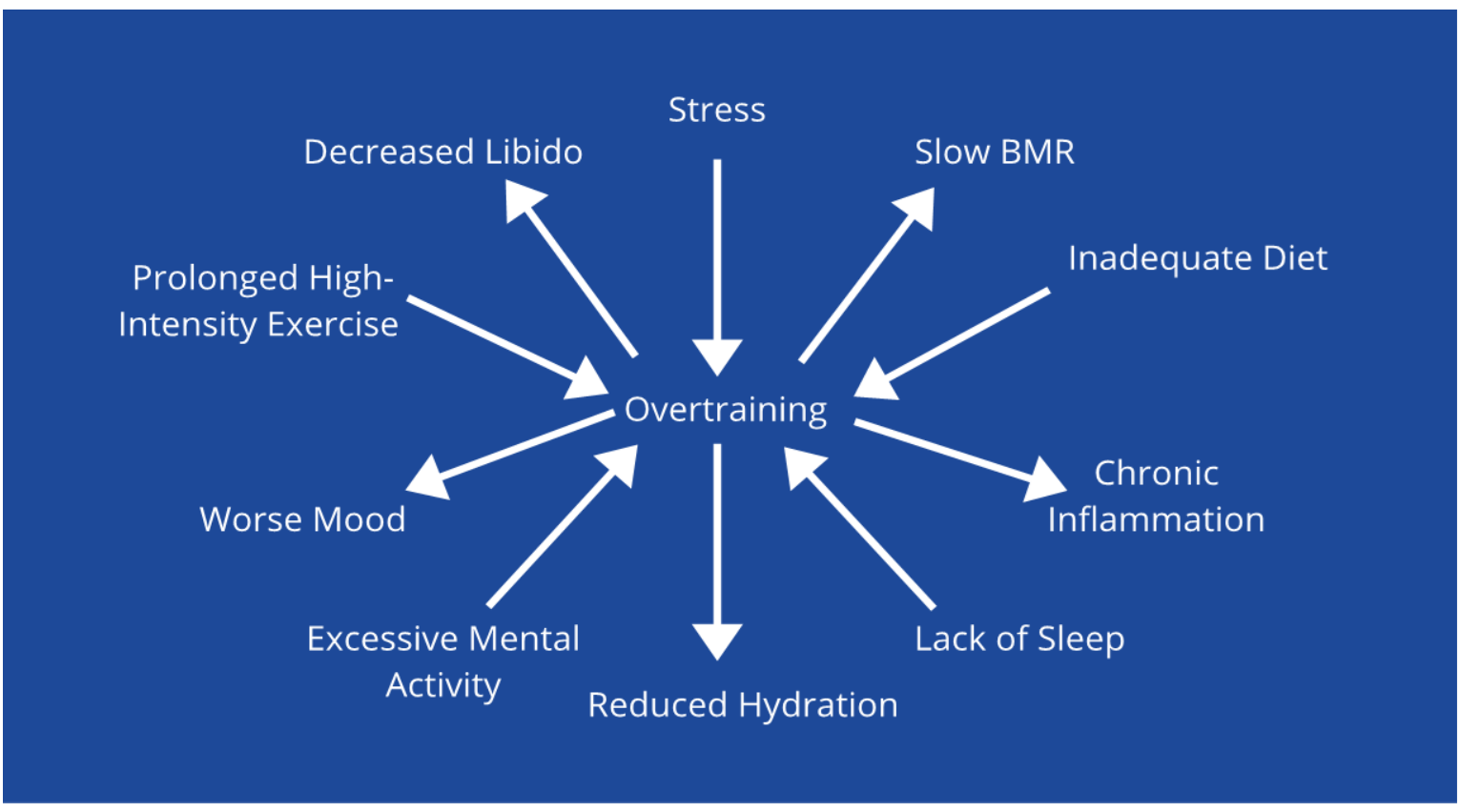

Figure 1. A comprehensive overview on the general causes and effects of overtraining. The figure was created based on the Cadegiani and Kater (2018) study.

\section{Critique}

The recruitment method likely involved self-selection bias and limits the validity of the findings. Furthermore, this study disregards metabolic adaptations. OTS consumed less calories than ATL, despite both groups being athletes. It is well-established that metabolism can change depending on diet/lifestyle, thus a slower metabolism observed in OTS may have been caused by previous undereating rather than from overtraining (Heilbronn et al., 2006). Future overtraining studies should record previous patterns of eating prior to participation in an effort to account for potential metabolic adaptions. Lastly, a rigorous recruitment criterion (i.e., randomized controlled setting) should be utilized to minimize potential participation bias.

\section{References}

Cadegiani, F. A., \& Kater, C. E. (2018). Body composition, metabolism, sleep, psychological and eating patterns of overtraining syndrome: Results of the EROS study (EROS-PROFILE). Journal of Sports Sciences, 36(16), 19021910. https://doi.org/10.1080/02640414.2018.1424498 
| 2021 | Volume 2 | Issue 1 | The Journal of Health, Sports, and Kinesiology |

Heilbronn, L. K., De Jonge, L., Frisard, M. I., DeLany, J. P., Larson-Meyer, D. E., Rood, J., ... \& Greenway, F. L. (2006). Effect of 6-month calorie restriction on biomarkers of longevity, metabolic adaptation, and oxidative stress in overweight individuals: a randomized controlled trial. JAMA, 295(13), 1539-1548.

https://doi.org/10.1001/jama.295.13.1539

\section{Journal of Health, Sports, \& Kinesiology | ISSN 2692-9864 | www.johsk.com}

